\title{
Implantes vestibulares
}

\section{Vestibular prosthesis}

\author{
Cristian Aedo Sánchez¹, Paul H. Délano Reyes ${ }^{2,3}$.
}

\begin{abstract}
RESUMEN
La lesión o hipofunción vestibular bilateral (HVB) es un cuadro clínico que provoca oscilopsias y desequilibrio incapacitante que se agrava en oscuridad. Algunas de las causas de HVB son el uso de drogas ototóxicas, enfermedad de Ménière bilateral, enfermedades autoinmunes y degeneración espinocerebelar. El manejo de esta discapacidad es complejo y muchas veces la rehabilitación no logra los objetivos deseados. Una de las alternativas terapéuticas futuras para la HVB y aún en plena fase experimental es el implante vestibular, cuyo funcionamiento en términos generales es similar al de un implante coclear. En esta revisión se analiza la génesis e historia del desarrollo de los implantes vestibulares, sus principales características y el futuro de su implementación.
\end{abstract}

Palabras clave: Lesión vestibular bilateral, enfermedad de Ménière, oscilopsias, desequilibrio, ototóxicos, implante vestibular.

\begin{abstract}
Bilateral vestibular loss (BVL) is a clinic syndrome that produces oscillopsias and disabling disequilibrium, especially in darkness. Some causes of BVL are the use of ototoxic drugs, bilateral Ménière disease, autoinmune ear disorders and spinocerebellar ataxia. The management of this disability is complex and many times the rehabilitation does not accomplish with the desired goals. One future therapeutic alternative for BVL and still in an experimental stage is the vestibular implant, whose overall functioning is similar to a cochlear implant. In this review we analyze the genesis and history of vestibular implant development, its main technical characteristics and the future of its implementation.

Key words: Bilateral vestibular loss, Ménière disease, oscillopsias, disequilibrium, ototoxic, vestibular implant.

1 Tecnólogo-Médico ORL. Magister Neurociencias (c), Programa de Fisiología y Biofísica, ICBM, Facultad de Medicina, Universidad de Chile.
\end{abstract}

2 Otorrinolaringólogo. Programa de Fisiología y Biofísica, ICBM, Facultad de Medicina, Universidad de Chile.

3 Médico Servicio Otorrinolaringología, Hospital Clínico Universidad de Chile. 


\section{INTRODUCCIÓN}

A diferencia de la sordera, ceguera o anosmia, no existe un término clínico exacto para definir la pérdida bilateral de la función vestibular y se utiliza el concepto de hipofunción vestibular bilateral (HVB) que engloba a lesiones parciales y completas de la función vestibular. Los pacientes con HVB presentan un cuadro clínico caracterizado por oscilopsias y desequilibrio incapacitante que se agrava en oscuridad ${ }^{1}$. Se ha descrito además que la HVB provoca pérdida de la memoria y orientación espacial con atrofia del hipocampo ${ }^{2}$. Por otra parte la presencia de vértigo y de hipoacusia es menos habitual, y depende de la presencia de asimetría en las lesiones vestibulares y de la etiología de la $\mathrm{HVB}^{3}$.

La alteración de la prueba de impulso cefálico, de la prueba de agudeza visual dinámica y de la prueba de Romberg sugieren la presencia de HVB $^{4,5}$. Además de estos tres test clínicos, la presencia de una prueba calórica con arreflexia vestibular bilateral o con una hipofunción con velocidades de la fase lenta del nistagmo poscalórico menores a $5 \%$ en forma bilateral confirman el diagnóstico de HVB ${ }^{6}$.

Dentro de la etiología de HVB encontramos el uso de drogas vestíbulo-tóxicas como la gentamicina ${ }^{7}$, enfermedades autoinmunes del oído interno, enfermedad de Ménière bilateral, meningitis, ataxia espino-cerebelar, síndrome de CANVAS (ataxia cerebelosa con neuropatía y arreflexia vestibular bilateral $)^{8}$, neurofibromatosis tipo 2 , enfermedad de Creutzfeld-Jacob y déficit de vitamina B12 entre otras. Es importante destacar, que a pesar de un detallado estudio etiológico, cerca del $50 \%$ de los pacientes queda un diagnóstico de HVB sin causa aparente ${ }^{3}$.

El manejo de la HVB incluye el tratamiento específico de la causa etiológica (ej.: corticoides en patologías autoinmunes) y la rehabilitación del equilibrio. Se describe que en el $80 \%$ de los casos el daño vestibular se mantiene en el tiempo y que la rehabilitación vestibular tiene resultados dispares $^{9,10}$. Recientemente, diversos grupos de investigación han estado desarrollando una neuroprótesis vestibular, cuyo funcionamiento en términos generales es similar al de un implante coclear, en la que un dispositivo electrónico implantado en forma crónica en el laberinto estimula con pulsos eléctricos a los nervios que inervan a las ampollas vestibulares ${ }^{11}$. En este artículo se revisa la génesis e historia del desarrollo de los implantes vestibulares, sus principales características y el futuro de su implementación.

\section{HISTORIA DE LOS IMPLANTES VESTIBULARES}

En el año 1964 Susuky y Cohen demostraron en monos y gatos que la estimulación eléctrica de los nervios ampulares puede producir un nistagmo a través de la vía del reflejo vestíbulo-ocular (VOR) ${ }^{12}$. Posteriormente, Gong y cols diseñaron una prótesis piezoeléctrica implantable con electrodos de platino, que detecta giros horizontales de la cabeza $( \pm 500 \%$ s) de cobayos y estimula eléctricamente a las fibras del nervio vestibular (entre 50 a 250 $\mathrm{Hz})^{13}$. Con estos implantes lograron gatillar un VOR por estimulación eléctrica de la ampolla del canal horizontal en cobayos. Además, en un grupo de animales ocluyeron el conducto semicircular lateral, y al colocar las prótesis lograron restablecer la función vestibular ${ }^{14}$. Los autores describen que tras la estimulación eléctrica del canal semicircular lateral, se generaba un VOR horizontal cada vez que el animal realizaba movimientos de tipo angular.

Por otra parte, el grupo de investigación del Departamento de Otorrinolaringología del Laboratorio de Neuro-ingeniería de John Hopkins, liderado por el Dr. Della Santina han desarrollado una prótesis vestibular multicanal ${ }^{15-17}$. Este implante consta de 3 ejes ortogonales entre sí con microsensores de giro, los cuales emulan la sensibilidad a la velocidad angular de cada uno de los canales semicirculares. La información de giro angular de los 3 ejes es codificada para estimular eléctricamente a través de tres electrodos ubicados en cada uno de los segmentos ampulares de cada canal semicircular y enviada hacia la rama correspondiente del nervio vestibular. Los procesadores y sensores están fijados externamente al cráneo y conectados a los electrodos implantados mediante un conector percutáneo. Este grupo de investigación ha probado estos implantes en chinchillas, a las que se les ha provocado una lesión vestibular a través de inyecciones de gentamicina intratimpánica ${ }^{15}$. Al implantar a chinchillas con HVB por gentamicina, 
lograron restablecer parcialmente el VOR ${ }^{15,18}$ y evidenciaron una mejora significativa en la actividad locomotora del animal ${ }^{19}$.

Della Santina y cols han evaluado el impacto de los implantes vestibulares en la función auditiva de chinchillas ${ }^{17}$. Midieron los potenciales evocados auditivos a 6 chinchillas antes y 3 a 9 semanas después de la cirugía del implante. Cuatro animales resultaron con pérdida auditiva severa mientras que las otras dos chinchillas mantuvieron sus niveles auditivos. Posteriormente, el paso de este grupo fue generar un acercamiento del implante vestibular al uso clínico en humanos. Para ello utilizaron como modelo al mono Rhesus (Macaca mulatta), un primate que posee un laberinto vestibular anatómicamente similar al humano ${ }^{20,21}$. Los resultados del reflejo VOR posimplante fueron mejores a los obtenidos en las chinchillas implantadas. En estos animales se observó un leve aumento de 5-10 dB sobre los umbrales del $A B R$ y de 2-14 dB en los umbrales de emisiones otoacústicas por producto de distorsión, en comparación a los resultados del animal previamente implantado, lo cual sugiere que los riesgos quirúrgicos de injuria coclear serian más bajos en primates que en roedores.

En el año 2011, un equipo de la Universidad de Washington liderado por Jay Rubinstein y James Phillips realizaron el primer implante vestibular unilateral en humanos a un hombre de 56 años que padece la enfermedad de Ménière ${ }^{22}$. A diferencia de un implante coclear, que utiliza un arreglo de electrodos insertados dentro de la cóclea para la estimulación del nervio auditivo, este implante vestibular utiliza 3 pequeños electrodos, los cuales son insertados en cada uno de los tres canales semicirculares para estimular el nervio vestibular. Los resultados de esta experiencia clínica han sido recientemente publicados en un grupo de cuatro pacientes con enfermedad de Ménière unilateral y refractaria a tratamiento con resultados aún preliminares ${ }^{23}$.

Por otra parte, Guyot y cols en el año 2011 realizaron una cirugía en la cual insertaron un implante coclear modificado en un sujeto con sordera profunda y arreflexia vestibular bilateral idiopática. Para ello utilizaron 11 electrodos intracocleares para la estimulación del nervio coclear y un electrodo para la estimulación vestibular, ubicado cerca del nervio vestibular inferior, en la profundidad del nicho de la ventana redonda. Uti- lizando pulsos repetidos de estimulación eléctrica observaron primero una respuesta nistágmica de gran amplitud la cual desaparece a los 27 minutos. Al detener la estimulación eléctrica el nistagmo se invierte, desapareciendo a los pocos minutos después de realizado el protocolo. A medida que se va repitiendo este protocolo, la velocidad de la componente lenta y la duración del nistagmo provocado es cada vez menor. Esta adaptación del sistema vestibular frente a la estimulación eléctrica podría ser de mucha importancia para el desarrollo de futuras neuroprótesis vestibulares ${ }^{24,25}$.

En relación a la cirugía para la colocación de un implante vestibular se necesita acceder a las ampollas de los canales semicirculares 0 a las fibras neurales que las inervan, y se han propuesto dos tipos de accesos diferentes: (i) acceso directo transmeatal a los nervios vestibulares y (ii) acceso transmastoideo a las ampollas vestibulares. En el primer caso se propone a través de un acceso transmeatal el fresado del nicho de la ventana redonda con exposición del nervio vestibular inferior y un fresado de la pared medial del epitímpano (removiendo cabeza y cuerpo de martillo y yunque) para acceder al nervio vestibular superior. El segundo abordaje propuesto para el acceso a las ampollas vestibulares se realiza a través de una mastoidectomía ${ }^{26}$. En este caso, después de la mastoidectomía es necesario realizar una pequeña fenestración en relación a cada ampolla vestibular. Se puede confirmar el funcionamiento del implante estimulando con pequeños pulsos eléctricos que provocan un nistagmo espontáneo en dirección al canal estimulado ${ }^{24,26}$. Las principales complicaciones quirúrgicas son dos: daño al nervio facial y sobre todo lesión auditiva que provoque una sordera sensorioneural posquirúrgica.

\section{CONCLUSIONES}

El avance de la investigación básico-clínica en el entendimiento de la fisiología vestibular, junto con el progreso de la electrónica y computación han permitido desarrollar la neuroprótesis vestibular que probablemente en el futuro cercano permitirá restablecer el equilibrio en el grupo de pacientes con HVB o con enfermedad de Ménière unilateral pero refractaria a tratamientos convencionales. 


\section{BIBLIOGRAFÍA}

1. Crawford J. Living without a balancing mechanism. N Engl J Med 1952; 246: 458-60.

2. Brandt T, Schautzer F, Hamilton DA, Brüning $R$, Markowitsch HJ, Kalla R, Darlington C, Smith P, StRuPP M. Vestibular loss causes hippocampal atrophy and impaired spatial memory in humans. Brain 2005; 128: 2732-41.

3. Zingler VC, Cnyrim C, Jahn K, Weintz E, Ferndacher J, Frenzel C, Brandt T, Strupp M. Causative factors and epidemiology of bilateral vestibulopathy in 255 patients. Ann Neurol 2007; 61: 524-32.

4. Petersen JA, Straumann D, Weber KP. Clinical diagnosis of bilateral vestibular loss: three simple bedside tests. Ther Adv Neurol Disord 2013; 6: 41-5.

5. Peña A. El examen vestibular abreviado, descripción, interpretación y análisis. Rev Otorrinolaringol Cir Cabeza Cuello 2011; 71 : 135-44.

6. Zingler VC, Weintz E, Jahn K, Huppert D, Cnyrim C, Brandt T, Strupp M. Causative factors, epidemiology, and follow-up of bilateral vestibulopathy. Ann N Y Acad Sci 2009; 1164: 505-8.

7. Minor LB. Gentamicin-induced bilateral vestibular hypofunction. JAMA 1998; 279: 541-4.

8. Szmulewicz DJ, Waterston JA, Halmagy GM, Mossman S, Chancellor aM, Mclean CA, Storey E. Sensory neuropathy as part of the cerebellar ataxia neuropathy vestibular areflexia syndrome. Neurology 2011; 76: 1903-10.

9. Zingler VC, Weintz E, Jahn K, Mike A, Huppert D, Rettinger N, Brandt T, Strupp M. Follow-up of vestibular function in bilateral vestibulopathy. $J$ Neurol Neurosurg Psychiatry 2008; 79: 284-8.

10. Riveros H, Correa CG, Anabalón Jl, Aranís C. Efectividad de la rehabilitación vestibular en una serie clínica. Rev Otorrinolaringol Cir Cabeza Cuello 2007; 67: 229-36.

11. Alzérreca E, Pardo J, Délano PH. Neuroprótesis en Otorrinolaringología: más allá del implante coclear. Rev Hosp Clín Univ Chile 2011; 22: 318-24.

12. Susuky JL, Cohen B. Head, eye, body and limb movements from semicircular canal nerves. Exp Neurol 1964; 10: 393-405.

13. Gong, W. and Merfeld DM. Prototype neural semicircular canal prosthesis using patterned electrical stimulation. Ann Biomed Eng 2000; 28: 572-81.

14. Gong, W. and Merfeld DM. System design and performance of a unilateral horizontal semicircular canal prosthesis. IEEE Trans Biomed Eng 2002; 49: 175-81.

15. Della Santina CC, Migliaccio aA, Patel aH. Electrical Stimulation to Restore Vestibular Function-Development of a 3-D Vestibular Prosthesis. Conf Proc IEEE Eng Med Bio Soc 2005; 7: 7380-5.

16. Della Santina CC, Migliaccio AA. A multichannel semicircular canal neural prosthesis using electrical stimulation to restore 3-d vestibular sensation. IEEE Trans Biomed Eng 2007; 54: 1016-30.

17. Tang S, Melvin TA, Della Santina CC. Effects of semicircular canal electrode implantation on hearing in chinchillas. Acta Otolaryngol 2009; 129: $481-6$.

18. Sun DQ, Rahman MA, Fridman G, Dai C, Chiang B, Della Santina CC. Chronic stimulation of the semicircular canals using a multichannel vestibular prosthesis: effects on locomotion and angular vestibulo-ocular reflex in chinchillas. Conf Proc IEEE Eng Med Biol Soc 2011; 2011: 3519-23.

19. Rahman MA, Dai C, Fridman GY, Davidovics NS, Chiang B, Ahn J, Hayden R, Melvin TA, Sun DQ, Hedjoudje A, Della Santina CC. Restoring the 3D vestibulo-ocular reflex via electrical stimulation: the Johns Hopkins multichannel vestibular prosthesis Project. Conf Proc IEEE Eng Med Biol Soc 2011; 3142-5.

20. Dal C, Fridman GY, Della Santina CC. Effects of vestibular prosthesis electrode implantation and stimulation on hearing in rhesus monkeys. Hear Res 2011; 227: 204-10.

21. Dai C, Fridman GY, Davinovics NS, Chiang B, Ahn JH, Della Santina CC. Effects of 3D vestibular sensation in rhesus monkeys using a multichannel vestibular prosthesis. Hear Res 2011; 281: 74-83.

22. Rubinstein J, Phillips J, Nie K. Clinical, Scientific and Regulatory Roadmap for a Human Vestibular Implant. 34 $4^{\text {th }}$ MidWinter Meeting of the Association for Research in Otolaryngology 2011; Abs. 266.

23. Phillips C, Defrancisci C, Ling L, Nie K, Nowack $A$, Phillips J0, Rubinstein JT. Postural responses 
to electrical stimulation of the vestibular end organs in human subjects. Exp Brain Res 2013 In Press.

24. Guyot JP, Sigrist A, Pelizzone M, Kos MI. Adaptation to steady-state electrical stimulation of the vestibular system in humans. Ann Otol Rhinol Laryngol 2011; 120: 143-9.

25. Rubinstein JT, Bierer S, Kaneko C, Ling L, Nie K, Oxford T; Newlands S, Santos F; Risi F, Abbas P, PHILLIPS J. Implantation of the semicircular canals with preservation of hearing and rotational sensitivity: a vestibular neurostimulator suitable for clinical research. Otol Neurotol 2012; 33: 789-96.

26. Van de Berg R, Guinand N, Guyot JP, Kingma $\mathrm{H}$, Storkroos RJ. The modified ampullar approach for vestibular implant surgery: feasibility and its first application in a human with a long-term vestibular loss. Front Neurol 2012; 3: 18. 